\title{
Prácticas inclusivas en la mediacion pedagógica: ¿estamos preparados?
}

\author{
Inclusive practices in the pedagogical mediation: are we prepared? \\ Maricruz Mejías Vindas ${ }^{1}$ \\ Fecha de recepción: 7-1-2019 \\ Fecha de aprobación: 17-9-2019
}

\begin{abstract}
Resumen
Este artículo científico es el resultado de una investigación de tipo mixta, cuyo propósito fue llevar a cabo un diagnóstico sobre necesidades de capacitación que tienen los docentes de la Dirección Regional de Occidente acerca de los temas relacionados con la Educación inclusiva. Para la recolección de datos, se tomaron en cuenta 36 escuelas de los diferentes circuitos institucionales, así como autoridades de la Dirección Regional que están relacionados directamente con el tema. Los instrumentos utilizados fueron cuestionarios, entrevistas, grupos focales y guías de observación. Entre los principales resultados encontrados, destaca la dificultad de los docentes para poner en práctica la inclusión en su práxis pedagógica por falta de capacitación y conocimiento en lo referente al tema. De ahí que se evidencia urgente crear espacios que le permitan a los docentes capacitarse en diferentes metodologías, lenguajes y técnicas que les permitan atender la diversidad del salón de clase.
\end{abstract}

Palabras claves: inclusión, necesidades, capacitación, mediación pedagógica, diversidad.

\begin{abstract}
This scientific article is the result of a mixed type of research whose purpose was to carry out a diagnosis on the training needs of teachers of the Regional Office of the West about the issues related to inclusive education. For the collection of data, 36 schools of the different school circuits were considered, as well as authorities of the Regional Office that are directly related to the subject. The instruments used were questionnaires, interviews, focus groups and observation guides. Among the main results found is the difficulty of teachers to implement inclusion in their pedagogical practice due to lack of training and knowledge regarding the subject. Hence, it is urgent to open spaces that allow teachers to train in different methodologies, languages and techniques that allow them to address the diversity of the classroom.
\end{abstract}

Key Words: inclusion, training needs, pedagogic mediation, diversity.

\footnotetext{
1 Magíster en Ciencias de la Educación. Docente e investigadora de la Universidad de Costa Rica, Sede de Occidente. Departamento de Educación. Costa Rica.

Correo electrónico: maricruz.mejias@ucr.ac.cr
} 


\section{Introducción}

Inclusión e integración son palabras ya bastante conocidas para los docentes de educación primaria por el contacto con directrices que emanan desde las oficinas centrales del Ministerio de Educación Pública. Sin embargo, la práctica de esas directrices dentro del salón de clase no logran los resultados esperados debido a que no existe armonía entre lo que se pretende lograr y la previa preparación de la persona que educa para lograrlo.

Este artículo expone parte de los resultados obtenidos en una investigación acerca de necesidades de capacitación en docentes de primaria sobre la temática relacionada con la educación inclusiva y la mediación pedagógica para lograr la atención a la diversidad en 36 centros educativos de la Dirección Regional de Occidente. Dicho estudio demuestra resultados dignos de analizar en lo que respecta el quehacer educativo y la necesidad de una atención igualitaria en el momento en que los estudiantes se preparan para interactuar con sus iguales y con sus docentes.

\section{Mediación pedagógica y su importancia}

Al hablar de mediación pedagógica nos referimos al proceso mediante el cual el docente guía al educando por medio de apoyos con el fin de que alcance el objetivo esperado. Entendemos por apoyos todo aquel material tecnológico, textos, instrucciones, cuestionarios que posibiliten la resolución satisfactoria de los diversos ejercicios o problemas que se le plantean.

Ramírez Prado (2009, pp. 4-6) analiza varios criterios que deberíamos tomar en cuenta en el momento de la mediación:

Intencionalidad: se refiere a la motivación para el aprendizaje. El estudiante descubre la intencionalidad de mediador y este lo incluye en la experiencia del aprendizaje

Trascendencia: se trata de ir más allá de lo que implica el aprendizaje en el aula.
Significado: buscar el por qué, para qué y cómo de todo lo que se aprende. Aprender a cuestionar y salir en la búsqueda de nuevas respuestas.

Sentimiento de competencia: despertar los deseos de superación. Construir nuevos aprendizajes por deseo propio.

Regulación de la conducta: se trata de mediar para que el alumno controle la impulsividad y busque el trabajo armonioso en el salón de clase. Comprender tanto los deberes como los derechos que tienen dentro de la clase.

Búsqueda de novedad y complejidad: presentar actividades en forma novedosa, no tener miedo a lo nuevo y propiciar la adaptación a experiencias diferentes.

Sentimiento de pertenencia: sentirse parte de un grupo social el cual tiene normas que podemos cambiar y mejorar.

Así, entendido la mediación se convierte en un proceso articulado, con intención y encaminado al logro de objetivos por y para el alumnado de manera que se obtenga un resultado a largo plazo reflejado en la capacidad de aquella persona para enfrentarse al mundo haciendo uso de una serie de habilidades

Estas acciones organizadas en la interacción pedagógica tiene la finalidad de promover y facilitar el proceso de aprendizaje de los estudiantes. Al respecto Díaz Barriga y Hernández Rojas (1999, p. 48) expresan que el docente es un organizador y mediador en el encuentro del alumnado con el conocimiento y su función primordial es la de orientar y guiar la actividad mental constructiva de su alumnado, a quienes proporcionará una ayuda pedagógica ajustada a sus competencias. De aquí se deriva que el docente tiene la gran responsabilidad de generar experiencias de aprendizaje que permitan al estudiante construir conocimiento. El docente debe despertar en sus estudiantes la curiosidad, la creatividad, el deseo de explorar otros mundos. Para ello, debe crear ambientes de aprendizaje interactivos, que favorezcan la autonomía, la reflexión y sobre todo la criticidad. El docente como mediador debe construir espacios que favorezcan el 
entendimiento mutuo y la tolerancia, donde la escuela se convierta para el niño en un lugar atractivo, que propicie aprendizajes significativos, al respecto Delors (1996, p.176) afirma que a los docentes les concierne de manera imperativa actualizar los conocimientos y las competencias, porque deben asumir la organización de su vida profesional de tal forma que estén en condición e incluso en la obligación de perfeccionar su arte, de aprovechar las experiencias realizadas en las distintas esferas de su vida económica, social y cultural.

Por esta razón, el docente debe estar en constante actualización, conocer el contexto de sus estudiantes y de la sociedad del conocimiento, respondiendo así a las exigencias de la educación y generar aprendizajes significativos mediante enfoques sociocríticos y reconstruccionistas, transformando la labor docente, convirtiéndose de esta manera la actividad en el aula, en un intercambio didáctico, un espacio de interacción, de motivación, donde la teoría y la práctica permitirán la construcción y reconstrucción de nuevos aprendizajes.

\subsection{Recursos tecnológicos en la mediación}

El docente del siglo XXI, debe incorporar en su mediación pedagógica el uso de nuevas tecnologías, debe ser percibido por su alumnado como un amigo y un modelo, como alguien que los escucha y los ayuda a desarrollarse. Debe ser investigador, innovador e incentivar a sus estudiantes a apropiarse de los conocimientos, valores y habilidades necesarias para aprender a conocer, aprender a hacer, aprender a ser y aprender a convivir como afirma Delors (1994, pp. 91-103).

Los recursos tecnológicos como herramientas pedagógicas responden a la capacidad del docente para adaptarse a nuevas experiencias que la ciencia le permite dando creatividad a sus lecciones y haciendo que diversas necesidades, que surgen del estudiantado, se vean desarrolladas con elementos que llaman su interés y al mismo tiempo le permiten tener una visión moderna de un tema en estudio. Hay una idea vygotskyana que apunta a que la imaginación y, en general, los procesos mentales se apoyan en la experiencia previa del sujeto desde la cual se toman los elementos necesarios para la creación de nuevos conocimientos. Por ejemplo, en una clase de Estudios Sociales antes de analizar el relieve de la provincia de Alajuela se puede compartir un video u observar juntos el mapa satelital para conocer de manera atractiva las irregularidades de esta, incluyendo ríos y cantidad de población.

\section{Ministerio de Educación Pública y los apoyos para la mediación docente}

El Ministerio de Educación Pública también ha incentivado el trabajo docente con el uso de recursos tecnológicos a través de su Programa Nacional de Tecnologías Móviles para la Educación (PNTM) Tecno@prender, el cual es una iniciativa orientada al desarrollo de la educación costarricense, por medio de la inclusión de las Tecnologías Digitales de la Información y la Comunicación (TDIC) en los procesos de enseñanza y aprendizaje como apoyo al currículo nacional favoreciendo el acceso y uso productivos de las tecnologías digitales en la comunidad educativa y estimulando el desarrollo de un pensamiento crítico y divergente en los estudiantes, mediante la promoción de la innovación en las prácticas de los docentes. Este programa fue creado en el 2015, con el fin de despertar en docentes la motivación hacia las nuevas tecnologías, además, de proporcionar en las instituciones educativas herramientas para utilizar en el salón de clase. Abarca todos los niveles de educación desde el materno hasta la educación diversificada.

\section{El docente como facilitador y mediador}

Uno de los conceptos que debe conocer un educador en su rol de mediador es el que Vygotski llama la zona de desarrollo próximo. Esta se refiere a "la distancia entre el nivel real del desarrollo determinado por la capacidad de aprender en forma independiente y el nivel de desarrollo potencial determinado por el aprendizaje con la colaboración de un compañero más capaz o con la guía de un adulto" (Vygotski, 1967, p. 28). Está relacionado con la mediación que lleva a cabo el docente con el fin de llevar a los estudiantes a su nivel de desarrollo potencial. Esto quiere decir que el docente se convierte en una especie de catalizador entre sus estudiantes y las experiencias hacia nuevos aprendizajes, sus experiencias y las relaciones entre sí. 
El término de mediación es de uso común en la bibliografía neovygotskyana (Frawley, 1999), ya que considera en esta perspectiva que la mente no aprehende de manera directa un saber o conocimiento del mundo exterior. Para que haya esa aprehensión se requieren mediaciones simbólicas, internas y sociales. Entre estas el lenguaje humano, el lenguaje que utiliza la computación, la escritura. No significa que la mediación se base únicamente en el lenguaje sino también cualquier tipo de símbolos, colores, marcas, imágenes. Esta perspectiva apunta a que, según Frawley (1999, pp. 186-187), es que las mediaciones contribuyen a que las representaciones externas se configuren como representaciones internas, así mismo, se conviertan en herramientas para la metaconciencia. Las representaciones externas facilitan la ejecución, porque muestran simultáneamente su información y su sistema de información, es decir, su representacionalidad, en cambio, las representaciones internas deben estipular esta última.

Gantus (2005, p. 54) afirma que el personal docente como facilitador cumple con las siguientes funciones:

El profesor, preferentemente, estructura el material, el medio o la situación de enseñanza, de modo que la interacción entre el estudiante y este ambiente organizado defina el camino a seguir o el objetivo a alcanzar. • Organiza un ambiente rico en estímulos donde se "dan" las estructuras que quiere enseñar. • Propone metas claras, apoya al estudiante en su elección. Desarrolla criterios para determinar si se llegó o no a la meta deseada. Luego de aceptadas, apoya el proceso de aprendizaje. • En la función de apoyo mantiene una posición permisiva y atenta.

De esta forma, se entiende que el fin primordial del docente es un acompañamiento al proceso de aprendizaje, que contemple diversas formas de aprender y que permita en todo momento la atención a la diversidad.

Dixon-Krauss (1996, p. 21), considera que la mediación en el proceso de instrucción está conformada por los planes y las acciones que el docente desarrolla durante este proceso. " $\mathrm{Si}$ el alumno necesita una ayuda, el docente se la ofrece, si el estudiante no tiene claro el concepto, el docente se lo define y lo puede remitir a otras fuentes: textos escolares, bases de datos, acceso a centros de documentación, consultas, on-line, libros u otros documentos de consulta". (DixonKrauss, 1996, p. 21).

Sin lugar a dudas, persiste entre los docentes un amplio abanico de actitudes con respecto a la mediación que debe realizar en el aula: algunos se centran en el cumplimiento de los contenidos que establece el plan de estudios; otros consideran que los momentos de la clase no son reales y se evocan a dar la lección. En algunos casos, el libro de texto es el único medio para el trabajo a realizar. Para otros, mantener un aula en completo silencio es la base del conocimiento. Al respecto, Perrenoud (2004, p. 5964) apunta que cada docente debe conocer una serie de competencias que le permitan hacer del aprendizaje algo más significativo, entre ellas:

1. Suscitar el deseo de aprender, explicitar la relación con el conocimiento, el sentido del trabajo escolar y desarrollar la capacidad de autoevaluación en el estudiante.

2. Negociar con el alumnado varias clases de reglas y obligaciones.

3. Ofrecer actividades de formación opcionales "a la carta".

4. Favorecer la definición de un proyecto personal del alumnado.

Según el autor, este tipo de actividades promueven un aprendizaje independiente, donde el estudiante toma conciencia del porqué de la escuela y su papel protagónico en el desarrollo de la misma.

Según Francis (2012, p. 41) "La función del docente en la escuela es servir de mediador para que se generen experiencias de aprendizaje". De esta manera la escuela es vista como un lugar donde el docente y sus estudiantes comparten, discuten, negocian y reconstruyen saberes construidos socialmente. "Lo que el alumno aprende no es una copia de lo que observa a su alrededor, sino el resultado de su propio pensamiento y razonamiento" (Kingler \& Vadillo 2001, p. 271). 
El papel del docente al ser mediador es organizar el medio social educativo, o sea, el ambiente escolar para hacer de la interacción escolar un momento que marca a sus estudiantes a través de la experiencia. Es por esto que Vygotsky no visualiza al docente como facilitador pues, al crear el camino más cómodo y fácil para la asimilación de los conocimientos, paraliza el acostumbramiento a pensar de manera independiente, le quita al niño esa preocupación. Podemos decir entonces que, de acuerdo a la mediación así será la oportunidad del estudiante para construir nuevos conocimientos siempre y cuando se le ofrezcan diversas maneras para llegar al aprendizaje. Esto significa que se desarrollen diversas acciones de mediación al presentar el contenido. "Comprender la mediación como una tarea docente no solo establece su función en la organización y regulación del medio escolar, también supone revisar las indicaciones pedagógicas referidas al cómo lograr que sus estudiantes hagan propias esas experiencias" (Parra, 2014, p. 42). De ahí que las herramientas de apoyo sean de gran utilidad, para garantizar variedad en el manejo de contenidos y a la vez despertar el interés en los estudiantes

\section{Educar para la vida}

El Ministerio de Educación Pública a través de las distintas reformas realizadas a los programas de estudio apuntan a un objetivo claro: educamos para la vida y dado que la vida se comparte con otros, educamos para la convivencia. Se pretende entonces que el educador costarricense replantee su forma de preparar su lección y además de los pilares filosóficos que ofrece la política educativa, tome en cuenta los cambios vertiginosos que ha tenido la sociedad que apunta a la inclusión y a la democracia según lo argumentan los planes de estudio.

Acerca de la metodología, el programa de estudio de Español I ciclo (2013, p .12) apunta que "el docente deberá aplicar estrategias didácticas y evaluativas que le permitan identificar los avances y dificultades individuales, para definir el tipo de acompañamiento que se requiere para así favorecer su progreso gradual en el desarrollo de las habilidades correspondientes a los objetivos curriculares, así como para la elaboración de un informe cualitativo de avance". Una práctica que toma en cuenta el aprendizaje que cada estudiante tiene, según su ritmo y capacidades. Igualmente, los programas solicitan a los docentes un enfoque renovado que responda a preguntas tales como: ¿cuáles son las principales necesidades de aprendizaje que hoy tienen nuestros estudiantes?, ¿de qué manera ellos aprenden mejor en la actualidad? y ¿cuáles son las formas de organización más apropiadas para fomentar esos aprendizajes? Agrega que en este proceso es de crucial importancia el aprovechamiento de las tecnologías de información y comunicación para estimular en los estudiantes un pensamiento creativo así como las habilidades de indagar, resolver problemas y trabajo en equipo que pueden actuar como "nuevas puntas de lanza para el logro de mayores niveles de calidad educativa" (Conare, 2011, p. 38)

\subsection{Perfil del estudiante que se espera formar}

Dentro del perfil del estudiante formado en ética estética y ciudadanía que propone el Ministerio de Educación Pública (MEP) en todos sus planes de estudio se pueden rescatar algunos enunciado que se ligan al trabajo que debe realizar el docente en el aula:

- Respeta y valora ideas distintas de las propias.

- Reconoce el diálogo como condición esencial de la convivencia social y de la superación de las diferencias.

- Desarrolla la responsabilidad de la libertad y autonomía personal respetando a sus compañeros.

- Capaz de trabajar en grupo y planificar actividades en conjunto, dialogando e intercambiando ideas y experiencias.

- Desarrolla la creatividad y la imaginación a través del juego, dramatizaciones, cuentos, invención de aventuras, viajes imaginarios, identificación de lugares ideales, entre otros. (Español, 2013, p. 14) 


\section{Mediación docente e inclusión}

La mediación docente, enfocada en la inclusión, parte de la filosofía de que todos los estudiantes pertenecen al grupo y todos pueden aprender en la vida normal de la escuela y de la comunidad. Se valora la diversidad, se cree que la diversidad refuerza la clase y ofrece mayores oportunidades de aprendizaje.

La pregunta será entonces: ¿cómo podemos hacer que los docentes tomen conciencia de que las diferencias de clase social, género, edad, capacidad, raza sean recursos para el aprendizaje? Seremos capaces de mostrar en el trabajo del aula la individualidad como un valor que permita tomar conciencia de que las diferencias del otro pueden ser un aprendizaje en cadena.

Susan Stainback (1999 menciona al profesor Robert Bath, profesor de Harvard, quien describe el valor de la diversidad "... las diferencias encierran grandes oportunidades de aprendizaje. Las diferencias constituyen un recurso gratuito abundante y renovable. Me gustaría que nuestra necesidad compulsiva de eliminar se sustituyese por un interés igualmente obsesivo por hacer uso de ellas para mejorar la enseñanza” (p. 34). Cada momento en el salón de clase debe ser una oportunidad para practicar la inclusión.

Algunas prácticas inclusivas que promueven una educación de calidad para todos son las siguientes:

a) Accesibilidad: consiste en eliminar todas aquellas barreras que impiden el aprendizaje y la participación de los estudiantes en las diferentes actividades. Todo centro educativo debe adaptar su entorno, programas y metodología para que todos los estudiantes, independientemente de su capacidad, logren avanzar, para ello deberán contar con apoyo adicional y seguimiento individualizado. El centro educativo debe ampliar sus fronteras y hacerse más accesible, para ello deberá promover espacios académicos extraescolares, para alumnado y familias. b) Cooperación y altas expectativas: toda la comunidad educativa, dirección, profesorado y familia tienen la responsabilidad y el compromiso de promover prácticas educativas que permitan el desarrollo de todos los estudiantes de acuerdo con sus capacidades, habilidades e intereses. Para ello, es indispensable fortalecer el trabajo en equipo, de esta manera el centro logrará que cada estudiante desarrolle al máximo sus capacidades. El aprendizaje desde la dialogicidad: se debe crear en toda institución una cultura de diálogo a través de grupos interactivos, que fomenten el compartir y la comunicación entre los distintos miembros de la comunidad educativa. Diversos estudios han determinado que una institución que involucra a la familia, especialmente aquellas del alumnado más vulnerable, redunda en un notable mejoramiento del rendimiento académico.

c) Utilización de recursos que generen el interés en el estudiantado: los recursos didácticos son herramientas que permiten desarrollar la motivación por aprender. Algunos teóricos del desarrollo humano, tales como Piaget, Bruner y Bandura afirman que, para que haya aprendizaje, debe existir emoción y precisamente este es el papel que debe jugar la mediación pedagógica. Los recursos que utilice el docente deben despertar el interés por aprender, además, facilitar las condiciones necesarias para que el alumno pueda llevar a cabo diferentes experiencias y saque de ellas el máximo provecho.

Existen también algunos aspectos referidos a la mediación pedagógica que pueden generar que en el aula se practique la inclusión. Stainback y Jackson (1995) aportan elementos tales como: las reglas dentro del salón donde se visualice el respeto y el tratamiento justo hacia cada una de las personas que conforman la comunidad estudiantil.

Otro aspecto, se refiere a la adaptación de la enseñanza al alumnado, de manera que se preste apoyo y asistencia para ayudarles a conseguir los objetivos curriculares adecuados. Esto se refiere a tomar en cuenta las distintas características y necesidades y logrando del currículo lo que está a su alcance, por medio de la adaptación. 
También se considera el apoyo en el aula. Si un estudiante requiere de apoyos curriculares o tecnológicos deben ser proporcionados en el aula ordinaria y no en un sistema especializado, aislado de sus iguales buscando satisfacer las necesidades en un ambiente natural. El trabajo en equipo, aprendizaje cooperativo y tutores por parte de los compañeros son una gran herramienta, para que el estudiante se sienta incluido.

Cuando en el salón de clase se requiere de la colaboración de personal experto, puede beneficiar no sólo al que lo solicita sino que puede colaborar al total. Por ejemplo, si un estudiante requiere de una especialista en lenguaje de señas, la atención en el aula permitirá que el resto del alumnado también conozca acerca del mismo y facilite la comunicación y el acompañamiento. Otros aspectos, como el fomento de la comprensión de las diferencias individuales, la flexibilidad y la capacitación son parte primordial de la mediación para la inclusión.

\section{Metodología}

En esta investigación se contó con la participación de 204 docentes, 183 mujeres y 21 hombres todos procedentes de la Dirección Regional de Occidente. Se tomó como muestra diferentes instituciones de los nueve circuitos que componen esta Dirección Regional con el propósito de lograr emitir criterios más válidos.
Además, se entrevistó a un representante del Equipo Itinerante y la Asesora Regional de Enseñanza Especial de la Dirección Regional de Occidente

Se elaboraron instrumentos para la recolección de datos (cuestionarios, guía para entrevistas no estructuradas, guía de observación y guía para grupos focales). Todos estos instrumentos fueron validados por una muestra de docentes y profesores universitarios de la Sede de Occidente.

Se trata de una investigación cualitativa. Con un tipo de muestra por conveniencia (permite seleccionar aquellos casos accesibles y próximos para el investigador y que acepten participar en la investigación de manera voluntaria).

\section{Descripción de resultados}

Con respecto a la mediación docente en la investigación se pueden describir algunos resultados de gran relevancia para la educación de nuestra región.

Se realizaron 36 observaciones donde la investigadora participó, mediando observacioes, en diversas lecciones de 80 minutos principalmente prácticas inclusivas en la mediación en grupos de Primero a Sexto Grado utilizando una tabla como la que se muestra a continuación:

\section{Tabla \#1}

Tabla de registro de observaciones

Fecha:

Nivel:

Escuela

\begin{tabular}{|l|l|l|l|}
\hline Lección observada & $\begin{array}{c}\text { Prácticas inclusivas } \\
\text { detectadas }\end{array}$ & Conclusiones de la observación & Otros aspectos relevantes \\
\hline & & & \\
& & & \\
& & & \\
\end{tabular}

Fuente: Elaboración Propia 
También se llevaron a cabo 5 grupos focales, en diferentes instituciones, con el objetivo de compartir experiencias de docentes relacionadas con esa necesidad de capacitación y el impacto que esta produce en el salón de clase. En estos grupos se trabajó la temática de los diferentes apoyos que los docentes ofrecen a sus estudiantes en el salón de clase, según lo establece el MEP. Para su ejecución se elaboraron las respectivas guías que, aunado a las grabaciones, proporcionaron información de mucho interés.

\section{Hallazgos principales con respecto a la mediación docente}

La investigación abarcó varios aspectos referidos a las necesidades de capacitación que tienen los docentes de educación primaria con respecto a la aplicación de apoyos educativos para responder a las políticas de la educación inclusiva, para efectos de este artículo se trabajará puntualmente con la labor que realizan los docentes en el salón de clase y en la institución educativa en general con respeto a la inclusión de los estudiantes, específicamente la mediación al desarrollar los diferentes contenidos de los programas dando énfasis al trato igualitario, vocabulario inclusivo e igualdad de oportunidades dentro del aula.

En las diferentes observaciones realizadas y la presentación preliminar del tema la mayoría de docentes accedieron a la visita en su grupo e inmediatamente señalaban a algunos estudiantes diciendo frases como.

"vea ese de allá tiene adecuación pero es por falta de límites en el hogar, es un vago”

"Yo tengo estos dos casitos que no avanzan, ni para atrás ni para adelante, posiblemente repitan porque no lograron el proceso de lectura"

"A este lo tengo sentado cerca de mí porque si no, imposible que avance"

Desde el inicio de la visita, se puede observar que los docentes relacionan inclusión con adecuación curricular y tienen muy bien identificado a los estudiantes "problema lo que nos permite evidenciar que existe una conceptualización errada de lo que realmente es inclusión.
Se puede afirmar que los docentes de primaria reciben poco apoyo, por parte de las autoridades, para el manejo de las adecuaciones con respecto al trato igualitario dentro del aula y por lo contrario utilizan etiquetas verbales como vago, desinteresado, lento, caso especial entre otros.

Otro aspecto que se pudo observar es el desarrollo de la clase donde los docentes en un $85 \%$ utilizan la forma tradicional, donde el docente es el protagonista siempre al frente y los estudiantes en pupitres ordenados en filas escuchando. Las estrategias de mediación se centran en la metodología tradicional:

- Uso de pizarra

- Uso de libro como recurso único de consulta

- Fichas didácticas que en su mayoría son copias de un libro

A pesar de que existen muchos estudios que demuestran que gran cantidad estudiantes pierden el interés o se mantiene interesados aproximadamente diez minutos en técnicas como esas, siguen siendo las preferidas. En los estudiantes puede notarse el trabajo y el avance individual, poca interacción entre estos y dispersión porque el trabajo permite que algunos terminen de manera rápida y otros hasta 20 minutos después que los primeros lo cual provoca desorden en el aula. En pocos casos (al menos un 15\% de los observados), se realizan actividades cooperativas, con el uso de algún tipo de tecnología y talleres que permiten al estudiante una mayor interacción y reflexión de lo que se realiza.

Dentro de las observaciones, un $5 \%$ de las docentes atendían niños diagnosticados con espectros u otras dificultades de aprendizaje, principalmente autistas, hiperactivida y asperger, también se visitó un aula donde asiste una niña con espina bífida. Entre los hallazgos más relevantes, en lo que respecta a la educación inclusiva, se pudo observar que estos estudiantes son ubicados en un lugar estratégico del aula donde, generalmente, no comparte con nadie excepto con el docente. Estos estudiantes trabajan con materiales diferentes al resto y, en algunas ocasiones, atendidos en lapsos por docentes de apoyo 
que ingresan a las lecciones del docente regular. Al preguntar acerca de esta práctica particular de aislar a los estudiantes los docentes responden:

"Es la única forma de que se concentre"

"Así evito que golpee a alguno de sus compañeros porque es muy agresivo"

"De esta forma evito que se me olvide atenderlo porque como su trabajo es diferentes a veces ya tengo rato de trabajar con el resto del grupo y a "S" (sic) no lo he atendido.

En el caso de la niña diagnosticada con espina bífida, se puede observar que usa una silla diferente, la docente explica que se trata de una necesidad que la niña tenía pero que la silla se la dieron desde que estaba en segundo, ahora está en sexto y ha crecido por lo que la silla ya no está cumpliendo la función que debe. Además, señala la docente que no se envió la mesa adecuada.

La utilización de recursos didácticos u otros tipos de apoyo que recomienda el MEP, para facilitar el proceso de aprendizaje de los estudiantes, dentro de los cuales destacan los apoyos personales, tecnológicos, organizativos y curriculares es un aspecto de gran importancia en la mediación pedagógica. Al preguntar a los docentes sobre los más utilizados se pudo comprobar:

El 87\% de docentes utiliza la atención individual como medio de apoyo personal dejando de lado la posibilidad del estudiante tutor o el trabajo en grupo como forma de fomentar la colaboración a estudiantes con debilidades de aprendizaje en alguna asignatura.

Los materiales tecnológicos no son accesibles a todos los docentes de la Región de Occidente, ya que en un 15\% de escuelas no cuentan con este tipo de recursos o los deben compartir entre 5 o más secciones. Igualmente, el concepto de tecnología se limita a un proyector o un televisor únicamente.

En lo que respecta a apoyos organizativos el 100\% de docentes cuentan solamente con la docente del servicio
SAFPA, dirigida a estudiantes con Adecuación Curricular y, así, atender la diversidad del aula.

En apoyos curriculares, en el 100\% de instituciones los programas educativos individuales son la única herramienta mencionada por los docentes.

\section{Cuadro No. 1 Apoyos que reciben los docentes para realizar una mediación pedagógica exitosa con los niños con necesidades educativas}

\begin{tabular}{|c|c|c|}
\hline Categoría & Cantidad & Porcentaje \\
\hline Apoyo del equipo itinerante & 47 & $23,03 \%$ \\
\hline $\begin{array}{l}\text { Capacitación del Comité de } \\
\text { apoyo }\end{array}$ & 103 & $50,49 \%$ \\
\hline $\begin{array}{l}\text { Ayuda para elaborar la } \\
\text { programación individual }\end{array}$ & 79 & $38,72 \%$ \\
\hline $\begin{array}{l}\text { Ideas innovadoras para realizar } \\
\text { una medición exitosa }\end{array}$ & 24 & $11,76 \%$ \\
\hline $\begin{array}{l}\text { Reuniones periódicas con el } \\
\text { personal para comentar ideas } \\
\text { innovadoras }\end{array}$ & 26 & $12,74 \%$ \\
\hline $\begin{array}{l}\text { Capacitaciones por parte del } \\
\text { Ministerio de Educación Pública }\end{array}$ & 35 & $17,15 \%$ \\
\hline $\begin{array}{l}\text { Apoyo en el aula de la docente } \\
\text { de apoyo } u \text { otro funcionario }\end{array}$ & 102 & $50 \%$ \\
\hline $\begin{array}{l}\text { Ayuda con la elaboración de } \\
\text { material didáctico para atender } \\
\text { al niño }\end{array}$ & 36 & $17,64 \%$ \\
\hline Ninguno & 36 & $17,64 \%$ \\
\hline
\end{tabular}

FUENTE: Elaboración propia a partir de los grupos focales

Al trabajar con los docentes, en los diferentes grupos focales, se pudo escuchar las principales preocupaciones que tienen con respecto al trabajo de aula y las diferentes dificultades en frases como las siguientes:

"El Ministerio no da acompañamiento a los docentes, les envía directrices a seguir y no nos capacita para realizar el trabajo, creen que el docente lo debe saber todo y no es así”

"Es demasiado el papeleo que realizamos en las escuelas. Cada año nos aumenta más y estamos perdiendo tiempo valioso con los niños por llenar fórmulas y solicitudes" 
"Nadie nos asesora... nadie nos explica... estamos solos entre tanta información. El padre de familia exige, el director y el asesor exige y nosotros ya no sabemos ni por donde ir aunque nuestro deseo es ayudar al estudiante"

"Existen tantas circulares que llegan al correo para nuevas directrices pero no tenemos tiempo de verlas"

"Este sistema por cascada para asesorar que usa el MEP se ha vuelto un teléfono chocho porque la información no llega o llega a la mitad y unos docentes terminan haciendo una cosa y otros otra, parece que no trabajamos para el mismo Ministerio"

\section{Cuadro No. 2 Mayores obstáculos encontrados para desarrollar el proceso educativo de manera fluida}

\begin{tabular}{|l|l|l|}
\hline Categoría & Cantidad & Porcentaje \\
\hline Falta de recursos & 95 & $46,56 \%$ \\
\hline Poca capacitación & 170 & $83,33 \%$ \\
\hline $\begin{array}{l}\text { Indisciplina de los } \\
\text { niños }\end{array}$ & 96 & $47,05 \%$ \\
\hline $\begin{array}{l}\text { G r u p o s } \\
\text { sobrecargados }\end{array}$ & 88 & $43,13 \%$ \\
\hline $\begin{array}{l}\text { Falta de apoyo } \\
\text { de los padres de } \\
\text { familia }\end{array}$ & 167 & $81,86 \%$ \\
\hline $\begin{array}{l}\text { La presión a la que } \\
\text { están sometidos en } \\
\text { la jornada escolar }\end{array}$ & 122 & $59,80 \%$ \\
\hline Otros & 52 & $25,49 \%$ \\
\hline
\end{tabular}

FUENTE: Elaboración propia a partir de entrevistas a docentes

La mediación pedagógica se ve obstaculizada por una serie de situaciones que, como lo refleja la tabla 2, impide a los docentes dedicar tiempo de calidad a los estudiantes debido a la de tareas extra que, según los entrevistados deben realizar. Además, la falta de recursos de las escuelas de la Dirección Regional de Occidente así cómo grupos sobrecargados dificultan que el momento más importante en la clase se desarrolle de la mejor manera.

\section{Conclusiones}

No cabe duda que todo lo que se logra visualizar es un problema de correcta capacitación y sensibilización hacia los conceptos de diversidad e inclusión, que inicia en el Ministerio de Educación Pública y repercute en el trabajo metodológico, que los docentes están llevando a cabo en sus salones de clase. Tal y como lo manifiesta Ramírez Prado (2009), la intencionalidad del aprender pierde su objetivo. La esencia del quehacer educativo, que se basa en la formación de personas independientes y de fomentar la escuela inclusiva, queda desplazada por el poco o nulo manejo de los conceptos esenciales transformadores de una sociedad igualitaria. Dentro de las conclusiones, se puede determinar que la mediación docente, lejos de favorecer la educación inclusiva en los casos estudiados, parece entenderse como una marcación en los estudiantes por aprender diferente. No se logra visualizar la diferencia como virtud sino como problema.

Muchas de las instituciones de educación primaria, de la Dirección Regional de Occidente, no cuentan con apoyos tales como tecnología para atender la diferencia de sus estudiantes y variar las técnicas para desarrollar sus lecciones.

La ausencia de capacitación que tienen los docentes en lo que se refiere a educación inclusiva provoca que visualicen a los estudiantes etiquetados con dificultades de aprendizaje como los únicos que requieren una atención diferente en las aulas. Se siguen utilizando conceptos errados acerca de la igualdad, integración e inclusión.

El docente dedica parte de su tiempo a aspectos administrativos y de papelería; tiempo que podría aprovechar para planear lecciones más amenas, que despierten el interés en sus estudiantes.

Sin actualización docente, es sumamente difícil lograr que en un circuito o región educativa exista armonía y consenso en temas tan obligatorios como lo es la inclusión y la integración por motivo de que se trata no sólo de la comprensión de estos sino del manejo que hago en el aula con respecto a la vivencia e interiorización del concepto. 
Para lograr en un 100\% una mediación pedagógica asertiva con respecto al tema de la educación inclusiva se hace necesario proponer escuelas que transformen desde la comunidad hasta lo interno de su estructura.

Se puede asegurar que, en la Región de Occidente, falta formación para llevar a cabo una verdadera práctica inclusiva en los salones de clases de primaria y hacer de la mediación pedagógica la principal herramienta para erradicar diferencias entre los estudiantes. Se requiere de capacitación, motivación y seguimiento en las instituciones educativas para que los apoyos requeridos se apliquen de manera efectiva.

\section{Bibliografía}

Delors, J. (1996). La Educación encierra un Tesoro. Informe a la UNESCO de la Comisión Internacional sobre educación para el Siglo XXI. París: UNESCO.

Dixon-Krauss, L. (1996). Vigotsky in the classroom. Mediatet Literacy. Instruction and Assesment, New York, Longman

Publishers USA.

Díaz-Barriga Arceo, F., \& Hernández Rojas, G. (2002). Estrategias docentes para un aprendizaje significativo. Una interpretación constructivista. $2^{\mathrm{a}}$. ed.) México: McGraw Hill.

Frawley, W., Adrian, A., \& Manuel, V. (1999). Barcelona: Paidós.

Gadner Howard (2001). La inteligencia reformulada. Barcelona: Paidós

Gantus, V. (2005,). La importancia de la mediación docente en los procesos de lectura de niños, adultos y jóvenes. Obtenido de http://www.feeye.uncu. edu.ar

Klinger, C. y Vadillo, G. (2001). Psicología Cognitiva. Estrategias de Prácticas Docentes. México: McGraw Hill.
Labarrete, A (2008) Bases conceptuales de la mediación y su importancia en la práctica pedagógica. SUMMA Obtenido desdehttps://dialnet.unirioja. es/descarga/articulo/2774206.pdf

Lobato Quesada, X. (2003). Diversidad y educación: taller de fortalecimiento: cuaderno de actividades. Mèxico, DF., Paidós

Ministerio de Educación Pública de la República de Costa Rica (2013). Programa de estudio de Español I ciclo. San José, MEP.

Programa Estado de la Nación 2017. Estado de la Educación Costarricense. San José: Servicios gráficos A.C.

Parra, K. N. (2014). El docente y el uso de la mediación en los procesos de enseñanza y aprendizaje. Revista de Investigación, 38(83), 155-180.

Perrenoud, P. (2004). Diez nuevas competencias para enseñar. Barcelona: Graó.

Ramírez Prado E (2009) La mediación en el proceso enseñanza- aprendizaje.https://archivos.csif.es/ archivos/andalucia/ensenanza/revistas/csicsif/ revista/pdf/Numero_14/ELENA_RAMIREZ_2. pdf

Salazar, F, S. (2012). El conocimiento pedagógico del contenido como modelo de mediación docente. San José, Costa Rica: Coordinación Educativa y Cultural. CECC/SICA.

Stainback, S., \& Stainback, W. (1999). Aulas inclusivas: un nuevo modo de enfocar y vivir el currículo (Vol. 79). Narcea Ediciones.

Stainback, S., \& Stainback, W. (1999). Aulas inclusivas: un nuevo modo de enfocar y vivir el currículo (Vol. 79). Narcea Ediciones.

Vygotsky, L. S. (1979). El desarrollo de los procesos psicológicos superiores. Buenos Aires: Grijalbo. 